\title{
Initiating telenephrology in the coronavirus disease (COVID) era: a tertiary care experience in India
}

\author{
Abhilash Chandra ${ }^{1}$, Namrata Rao $^{1}$, Divya Srivastava $^{2}$ \\ ${ }^{1}$ Department of Nephrology, Dr. Ram Manohar Lohia Institute of Medical Sciences, Lucknow, India \\ ${ }^{2}$ Department of Anesthesiology, Sanjay Gandhi Postgraduate Institute of Medical Sciences, Lucknow, India
}

Telemedicine is a broad term that encompasses the use of electronic information and communication technologies to provide healthcare remotely [1]. It involves the use of messages, telephone calls, video calls, and e-mail. Utilization of telemedicine began in the 1960s, but its use has not gained much ground in India. However, with the onset of the coronavirus disease 2019 (COVID-19) pandemic along with its restrictions on movement and financial constraints, it became imperative to start offering consults electronically.

Telemedicine offers remote consults, thereby saving time and travel cost and the associated inconvenience and pollution. It aids in timely referrals and prevents direct social interaction in cramped outpatient departments (OPDs). With most nephrologists located in urban areas, it provides important healthcare opportunities for the rural population.

Telemedicine, through video conferencing, was started in the month of May 2020 at our tertiary care hospital. It is handled by a trained clinic manager. Time and again relevant telephone numbers are advertised in national newspapers. The patients are required to call on these numbers to obtain an appointment for the respective department. They are given a fixed date and time at which they are expected to be near a video call enabled phone number provided by them. At the time of the slated appointment, a video call is made to the patient. If there is no response, an audio call is subsequently made. After a live two-way conversation, a prescription is forwarded to the patient in a portable document format (pdf). At the time of discussion with the patient, one consultant and one resident are present. Nephrology consultation is currently being offered once a week with the potential to further increase in frequency depending on the response to telemedicine services. In total, 15 to 25 consultations are completed in a single sitting. If needed, appointments for further follow-up are scheduled. Full responsibility for ethical considerations including privacy, informed consent, and confidentiality of the consults is undertaken by the hospital administration. Apart from this, digital consults are also being provided on WhatsApp (WhatsApp LLC) or via e-mail/message directly by the respective consultants.

Although good internet speed is maintained by the hospital (20-30 Mbps), disruptions due to poor signal/ technical issues on the patient's side are occasionally seen. As this is altogether new to the patients, sometimes they have difficulty communicating about accessibility problems along with minor camera focus issues. Also, the

Received: July 12, 2020; Revised: September 16, 2020; Accepted: October 13, 2020

Editor: Young-Ki Lee, Hallym University, Seoul, Republic of Korea

Correspondence: Abhilash Chandra

Department of Nephrology, Dr. Ram Manohar Lohia Institute of Medical Sciences, Vibhuti Khand, Gomti Nagar, Lucknow 226010, India. E-mail: acn393@gmail.com

ORCID: https://orcid.org/0000-0002-9055-4351

Copyright @ 2021 The Korean Society of Nephrology

(a) This is an Open Access article distributed under the terms of the Creative Commons Attribution Non-Commercial and No Derivatives License (http:// creativecommons.org/licenses/by-nc-nd/4.0/) which permits unrestricted non-commercial use, distribution of the material without any modifications, and reproduction in any medium, provided the original works properly cited. 
emotional connection, which is crucial in explaining the severity of the disease or offering a new therapy like dialysis initiation, is often missing. This arrangement is not meant for sicker patients for whom an in-person visit to the hospital emergency department is the only option. Interdepartmental referrals are not possible in the same sitting and a new appointment for a later date is given. The number of patients seen is limited and dramatically decreased compared to regular OPDs, which leaves a wide gap in unmet need.

Compilation of the data (April to June 2020) revealed that $86 \%$ of patients seen through this setup were regular follow-ups (Table 1). Of these $69 \%$ of patients were chronic kidney disease patients. Most of these were either advised to continue the same treatment or were offered minor changes. The second most frequent were consults offered to patients with primary/secondary glomerulonephritis on active immunosuppression who were not able to visit OPDs because of travel constraints. The age of the patients ranged from 18 to 60 years.

Despite these limitations, telemedicine remains a viable option for patients in remote or rural areas or otherwise not able to attend in-person appointments who may continue to benefit from virtual consultation even in a post-COVID era. Because nephrological evaluation relies

Table 1. Nephrology telemedicine consultations

\begin{tabular}{lc}
\hline Variable & Data $(\mathrm{n}=225)$ \\
\hline Follow-up & $193(85.8)$ \\
New patients & $32(14.2)$ \\
Sex, male:female & $162(72.0): 63(28.0)$ \\
Distribution by diagnosis & \\
Chronic kidney disease & $133(59.1)$ \\
$\quad$ eGFR (mL/min/1.73 $\left.\mathrm{m}^{2}\right)$ & \\
$>60$ & 18 \\
$45-60$ & 23 \\
$30-44$ & 33 \\
$15-29$ & 36 \\
$<15$ & 23 \\
Acute kidney injury & $12(5.3)$ \\
Nephrotic syndrome & $30(13.3)$ \\
Glomerulonephritis & $32(14.2)$ \\
Postrenal transplant & $18(8.0)$
\end{tabular}

Data are presented as number (\%) or number only. eGFR, estimated glomerular filtration rate by Chronic Kidney DiseaseEpidemiology Collaboration. primarily on history and lab workup, an electronic setup might be sufficient, though it does not completely replace a thorough examination. Telemedicine is better suited to patients who have already been evaluated locally or are in a regular follow-up in the hospital OPD. It should aim to provide effective services ensuring safe and timely solutions to patients' problems. There should also be a feedback system in place to verify the patient satisfaction [2]. The current scenario demands integration of this relatively new communication system into the existing doctor-patient interactive relationship. Other than being a tool for effective patient management, its utility can be extended to impart knowledge via educational and awareness interventions, which may benefit the community as a whole.

\section{Conflicts of interest}

All authors have no conflicts of interest to declare.

\section{Authors' contributions}

Conceptualization: AC, NR

Data curation: All authors

Investigation: $\mathrm{AC}, \mathrm{NR}$

Project administration: All authors

Writing-original draft: All authors

Writing-review \& editing: All authors

All authors read and approved the final manuscript.

\section{ORCID}

Abhilash Chandra, https://orcid.org/0000-0002-9055-4351 Namrata Rao, https://orcid.org/0000-0002-5733-4218 Divya Srivastava, https://orcid.org/0000-0003-1050-8521

\section{References}

1. World Health Organization (WHO). A health telematics policy in support of WHO's health-for-all strategy for global health development. Paper presented at: WHO Group Consultation on Health Telematics; 1997 Dec 11-16; Geneva, Switzerland.

2. Whitten P, Love B. Patient and provider satisfaction with the use of telemedicine: overview and rationale for cautious enthusiasm. $J$ Postgrad Med 2005;51:294-300. 